\title{
Neurosurgical digital teaching in low-middle income countries: beyond the frontiers of traditional education
}

\author{
Federico Nicolosi, MD, ${ }^{1}$ Zefferino Rossini, MD, ${ }^{1}$ Ismail Zaed, ${ }^{2}$ Angelos G. Kolias, MD, ${ }^{3,4}$ \\ Maurizio Fornari, MD, ${ }^{1}$ and Franco Servadei, MD ${ }^{2,5}$ \\ 'Department of Neurosurgery, Humanitas Clinical and Research Center, Rozzano (MI); ${ }^{2 H u m a n i t a s ~ U n i v e r s i t y, ~ V i a ~ R i t a ~ L e v i ~}$ \\ Montalcini, Pieve Emanuele (MI), Italy; ${ }^{3}$ Division of Neurosurgery, Department of Clinical Neurosciences, Addenbrooke's Hospital \\ \& University of Cambridge; ${ }^{2} \mathrm{NIHR}$ Global Health Research Group on Neurotrauma, University of Cambridge, Cambridge, United \\ Kingdom; and ${ }^{5}$ World Federation of Neurosurgical Societies, Nyon, Switzerland
}

\begin{abstract}
OBJECTIVE Neurosurgical training is usually based on traditional sources of education, such as papers, books, direct surgical experience, and cadaveric hands-on courses. In low-middle income countries, standard education programs are often unavailable, mainly owing to the lack of human and economic resources. Introducing digital platforms in these settings could be an alternative solution for bridging the gap between Western and poor countries in neurosurgical knowledge.

METHODS The authors identified from the Internet the main digital platforms that could easily be adopted in low-middle income countries. They selected free/low-cost mobile content with high educational impact.

RESULTS The platforms that were identified as fulfilling the characteristics described above are WFNS Young Neurosurgeons Forum Stream, Brainbook, NeuroMind, UpSurgeOn, The Neurosurgical Atlas, Touch surgery, The 100 UCLA Subjects in Neurosurgery, Neurosurgery Survival Guide, EANS (European Association of Neurosurgical Societies) Academy, Neurosurgical.TV, 3D Neuroanatomy, The Rhoton Collection, and Hinari. These platforms consist of webinars, 3D interactive neuroanatomy and neurosurgery content, videos, and e-learning programs supported by neurosurgical associations or journals.
\end{abstract}

CONCLUSIONS Digital education is an emerging tool for contributing to the spread of information in the neurosurgical community. The continuous improvement in the quality of content will rapidly increase the scientific validity of digital programs. In conclusion, the fast and easy access to digital resources could contribute to promote neurosurgical education in countries with limited facilities.

https://thejns.org/doi/abs/10.3171/2018.7.FOCUS18288

KEYWORDS global neurosurgery; LMICs; digital neurosurgical learning; e-learning; simulation; networking

$\mathrm{N}$ EUROSURGICAL education largely consists of training gained from reading books, attending medical congresses, and practicing in an operating theater or a cadaver lab. All these activities need a level of financial support that may be difficult to achieve for neurosurgeons in low-middle income countries (LMICs). Many digital tools and technological resources, designed to support different skills of neurosurgical training, attempt to provide support that complements standard education, offering 3D interactive simulations, procedural animations, webinars, and scientific advertisements. Although these formats can- not be considered a replacement for standard resources, their global diffusion through the Internet suggests a new trend in providing education and knowledge, regardless of the country's wealth. Considering that many digital teaching tools are entirely free and available online, they may represent a valid support for neurosurgical education as they have the potential to bridge the gap between surgeons from Western countries and colleagues from LMICs.

Although surgical skills must ultimately be taught and improved in the operating theater, we believe that some digital tools showing neurosurgical practice or simulating

ABBREVIATIONS EANS = European Association of Neurosurgical Societies; ETV = endoscopic third ventriculostomy; FIENS = Foundation for International Education in Neurological Surgery; LMIC = low-middle income country; NETS = Neurosurgery Education and Training School; NREF = Neurosurgery Research and Education Foundation; UN = United Nations; WFNS = World Federation of Neurosurgical Societies.

SUBMITTED June 1, 2018. ACCEPTED July 17, 2018.

INCLUDE WHEN CITING DOI: 10.3171/2018.7.FOCUS18288. 
surgical scenarios can provide an accessible way to reduce the gap in neurosurgical education between surgeons from countries of differing wealth and to create a structured online curriculum. ${ }^{10}$

Education tools to be adopted in countries with limited facilities should be not only easily accessible and low cost, but also regularly updated, oriented to surgical practice, and of scientific validity (supported by international neurosurgical societies and/or journals with high impact factors).

Here we describe the experience of some neurosurgical education programs supported by digital technology in LMICs, focusing on the main digital neurosurgical education platforms that present the characteristics described above.

\section{Methods}

We searched using PubMed (MEDLINE) and Google (web search) as well as the Apple App Store and Google Play for the following items: current neurosurgical education programs, comparing the training programs in Western countries with some programs carried out in countries with limited facilities; experiences of digital neurosurgical education addressed toward LMICs; neurosurgical digital platforms, including only those free or low-cost, mobile sources providing 3D content, interactive decision-making support, online webinars, and classes with expert specialists.

\section{Results}

\section{Current Traditional Neurosurgical Education Programs}

Neurosurgical training programs may differ significantly between countries. This is obviously due to many factors, such as available funds, internal organization, different epidemiology of diseases, and the distribution of primary, secondary, and tertiary centers. In Western countries, neurosurgical training programs are defined by a structured curriculum, mainly based on a combination of theoretical knowledge, surgical activities, research, and fellowships. Theoretical and practical aspects of neurosurgical training are highly variable throughout European countries. In a recent survey submitted to European neurosurgical residents between June 2014 and March 2015, satisfaction rates with practical aspects of training such as hands-on surgical experience (overall 73.9\%), microsurgical training (52.5\%), and simulator training (13.4\%), among others, were highly country dependent. In general, $89.1 \%$ of European residents carried out the first surgical procedure under supervision within the 1st year of training. Supervised lumbar/cervical spine surgeries were performed by $78.2 \%$ and $17.9 \%$ of European residents within 12 and 24 months of training, respectively, and $54.6 \%$ of European residents operate a cranial case within the first 36 months of training. ${ }^{21}$

In the United States, neurosurgical education comprises a 6-year residency as defined by the Accreditation Council for Graduate Medical Education (a 12-month internship followed by 60 months devoted to the neurological sciences: 36 in clinical neurosurgery, 12 months as chief resident, and 3-6 months in clinical neurology, neuropathology, neurology, neuroradiology, specialized neurosurgical training, and research). To maintain certification, a 10-year cycle-based maintenance of competencies is expected. ${ }^{20}$

Despite the difficulty of carrying out such a program in developing countries, ongoing strategies to improve international neurosurgical care have largely relied on visits made by individual surgeons to regions of LMICs in need. Examples of neurosurgical education experience in LMICs have been provided by the Foundation for International Education in Neurological Surgery (FIENS) (http:// www.fiens.org/), which has been training neurosurgeons around the world since 1969 in such projects as the Tanzania Neurosurgery Project, the Duke East Africa Neurosurgery Program, and the Cambodia Neurosurgery Support Project. CURE International and the International Federation for Spina Bifida and Hydrocephalus have supported the creation of iPATH. This program, which focuses on the treatment of hydrocephalus in 9 developing countries in Africa, has already trained 10 neurosurgeons. ${ }^{19}$

Another experience is Madaktari Africa, a training program that was started in Tanzania in 2006 and is based on the train-forward model. The aim of this project was to locally teach emergency neurosurgical procedures. It has led to an increase in the number of neurosurgical procedures performed by Tanzanian neurosurgeons from $44 \%$ in 2005 to approximately $86 \%$ in 2010 and a reduction in the rate of postoperative complications.?

In addition, the Neurosurgery Education and Development (NED) Foundation and FIENS introduced in 2006 a mobile teaching project for endoscopic third ventriculostomy (ETV) procedures and, subsequently, a program to promote the development of a permanent service of neurosurgery in Kenya and Zanzibar. Thanks to their first teaching program, almost 400 infants with hydrocephalus have been treated with ETV, with a success rate of $51 \%$, during the first 7 years. The second program, started in 2009 and carried on by 49 volunteer neurosurgeons, allowed 360 non-endoscopic neurosurgical procedures to be performed to treat hydrocephalus. ${ }^{15}$

Other education experiences, supported by the World Federation of Neurosurgical Societies (WFNS), like that of WFNS-Rabat Training Center (WFNS-RTC), Recife WFNS Reference Training Center (Brazil), and "Africa 100," have been already described elsewhere. ${ }^{19}$

\section{Current Experiences With Digital Neurosurgical Education Directed Toward LMICs}

The main projects dealing with the application of digital technology to improve education in the field of neurosurgery in LMICs use e-learning platforms. ${ }^{4,22}$ E-learning has been defined as "an approach to teaching and learning, representing all or part of the educational model applied, that is based on the use of electronic media and devices as tools for improving access to training, communication and interaction and that facilitates the adoption of new ways of understanding and developing learning." ${ }^{4}$

In 2014, Jotwani et al. described the experience of Neurosurgery Education and Training School (NETS), an e-learning platform founded by a multidisciplinary team of specialists as a "free-access, online, multi-authored, 
multi-institutional database," providing connectivity to neurosurgeons across the world. It contains educational material from downloadable presentations and 3D-animation videos to social networking and forums. The NETS elearning platform is a composite effort of the Department of Neurosurgery, All India Institute of Medical Sciences (AIIMS), New Delhi, and the Department of Computer Science and Engineering, Indian Institute of Technology (IIT), Delhi. The human resources involved are the content providers, data operators, system managers, 3D animators and graphic designers, the supporting management staff and a team of neurosurgeons, technologists, and application specialists who integrate the contents at the Neurosurgery Animation, Graphics and Video Editing Laboratory (NAGVEL). Content is evaluated by a members of a faculty advisory board (neurosurgeons from India and Germany) and technologists from IIT-Delhi. ${ }^{9}$

In April 2010, neurosurgery residents at the Korle Bu Teaching Hospital, in Accra, Ghana (http://wfns-neuroed. org), directed the first WFNS pilot project of a structured online course. The initial pilot project consisted of clinical modules, involving lectures in neuroanatomy and clinical case scenarios. The course lasted for 12 weeks. A second course started in September 2010 and covered the topic of "Introduction to Scientific Writing," in which they incorporated assignments with prompt responses, maintained discussion through online forums, and received positive feedback from the participants. ${ }^{2}$

Organizations such as the Ptolemy Project (Canada) and the School for Surgeons (Ireland) have employed e-learning tools to supplement the surgical training and advance the careers of surgeons in LMICs. The Ptolemy project (http://www.ptolemy.ca/) is an online resource model that allows African physicians to access journal articles for their clinical and educational use. ${ }^{5}$ Online courses administered through a stable virtual classroom platform such as Moodle (www.moodle.org) or WebCT (www.webct.com) allow for an in-depth check of content delivery, including material and time of the lectures.
Finally, the Weill Cornell Tanzania Neurosurgery Project (www.weillcornellbrainandspine.org/tanzania) was started in 2008 by Roger Härtl, MD, in collaboration with FIENS. The program focuses on Bugando Medical Center in Mwanza, Northern Tanzania, and Muhimbili Orthopedic Institute in Dar es Salaam. The program consists of 6 components, including a weekly Skype call and multidisciplinary meetings. ${ }^{3}$

\section{Neurosurgical Digital Platforms}

As universally agreed, the gold standard for neurosurgical education is a strong theoretical base accompanied by intense practice, which can be obtained by teaching in the operating theaters and with cadaver lab activity. Nevertheless, the wide improvement of digital technologies observed in the last decade created a new fascinating array of supportive tools feasible to be integrated into a standard curriculum. . $^{111,12,18,22}$

Some of these new technologies and applications have been designed to support different skills of neurosurgical training, including 3D imagination, procedural automatisms, decision-making, scientific updating and networking. Most of them are freely available online.

\section{WFNS Young Neurosurgeons Forum Stream}

YNF Stream (www.upsurgeon.com/apps) is an online channel through which the Young Neurosurgeons Forum, a committee of the WFNS, posts educational content and neurosurgical events to favor learning and networking in neurosurgery. The Stream is hosted within the neurosurgery-focused educational apps of the AppSurgeOn series, created by UpSurgeOn (Fig. 1).

\section{Brainbook}

Brainbook (http://www.realbrainbook.co.uk) is a United Kingdom (UK)-based platform created in order to facilitate neurosurgical case-based-learning, with the ambition to cover every aspect, from patient and family experi-
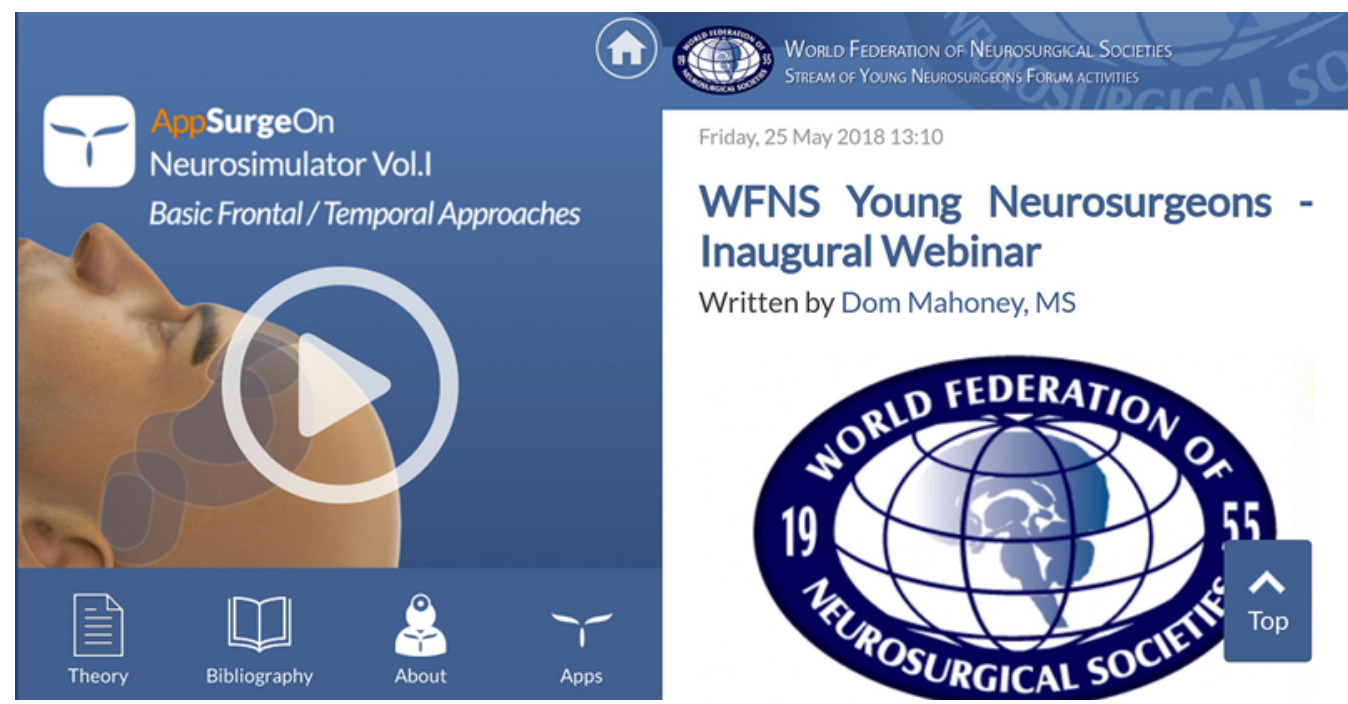

Friday, 25 May 2018 13:10

\section{WFNS Young Neurosurgeons - Inaugural Webinar}

Written by Dom Mahoney, MS

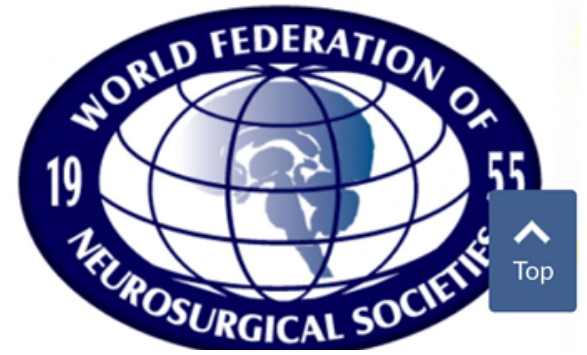

FIG. 1. Screenshot from the app UpSurgeOn (http://www.upsurgeon.com/apps) featuring the WFNS-Young Neurosurgeons Forum Stream. Copyright 2017 UpSurgeOn/WFNS Young Neurosurgeons Forum. Used with permission. 


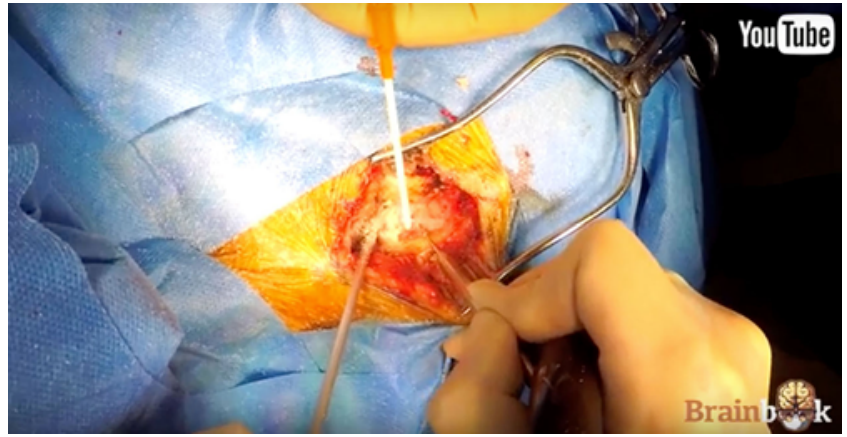

FIG. 2. Screenshot of video from Brainbook on YouTube. https://www. youtube.com/channel/UCnbPqck6c4yydPKVKOFaf6w. Copyright Brainbook. Used with permission.

ences to the more specific technicalities of the procedures. This is achieved through the use of illustrations, GoPro videos, photographs, and brain scans (Fig. 2).

\section{NeuroMind}

NeuroMind (http://www.neuromind.cc) is an app/ website that offers classifications and grading systems to support clinical decision-making in neurosurgery. It is officially supported by Surgical Neurology International and the European Association of Neurosurgical Societies (EANS).

\section{UpSurgeOn}

UpSurgeOn (www.upsurgeon.com) consists of a library of content made by a team of young neurosurgeons, developers, and digital artists. It offers apps and theoretical articles. AppSurgeOn, the app series, has been designed to improve theoretical knowledge of neuroanatomy and neurosurgical procedures and it is supported by the Young Neurosurgeons Forum of the WFNS. Currently it contains 3 apps (Fig. 3).

\section{The Neurosurgical Atlas}

The Neurosurgical Atlas (www.neurosurgicalatlas.com) is a collection of texts, intraoperative images, illustrations, and videos. The ultimate mission of this platform is to create an educational and clinical support for trainees and neurosurgeons of any level (Fig. 4).

\section{Touch Surgery}

Touch surgery (www.touchsurgery.com) is a free app series that simulates over 100 procedures of all the surgical specialties. It offers step-by-step interactive animations of specific procedures. It also allows an evaluation of the trainee's performance. Currently it contains 9 neurosurgical interactive animations (Fig. 5).

\section{The 100 UCLA Subjects in Neurosurgery}

This platform (www.neurosurgery.ucla.edu/subjects-inneurosurgery) constitutes a selection of a 100 relevant topics developed by the UCLA Department of Neurosurgery as an educational tool for residents of all training levels. The platform allows access to the radiology, pathology, anatomy, and ethics conferences organized by the department faculty.

\section{Neurosurgery Survival Guide}

Neurosurgery Survival Guide (NSG) is a neurosurgery

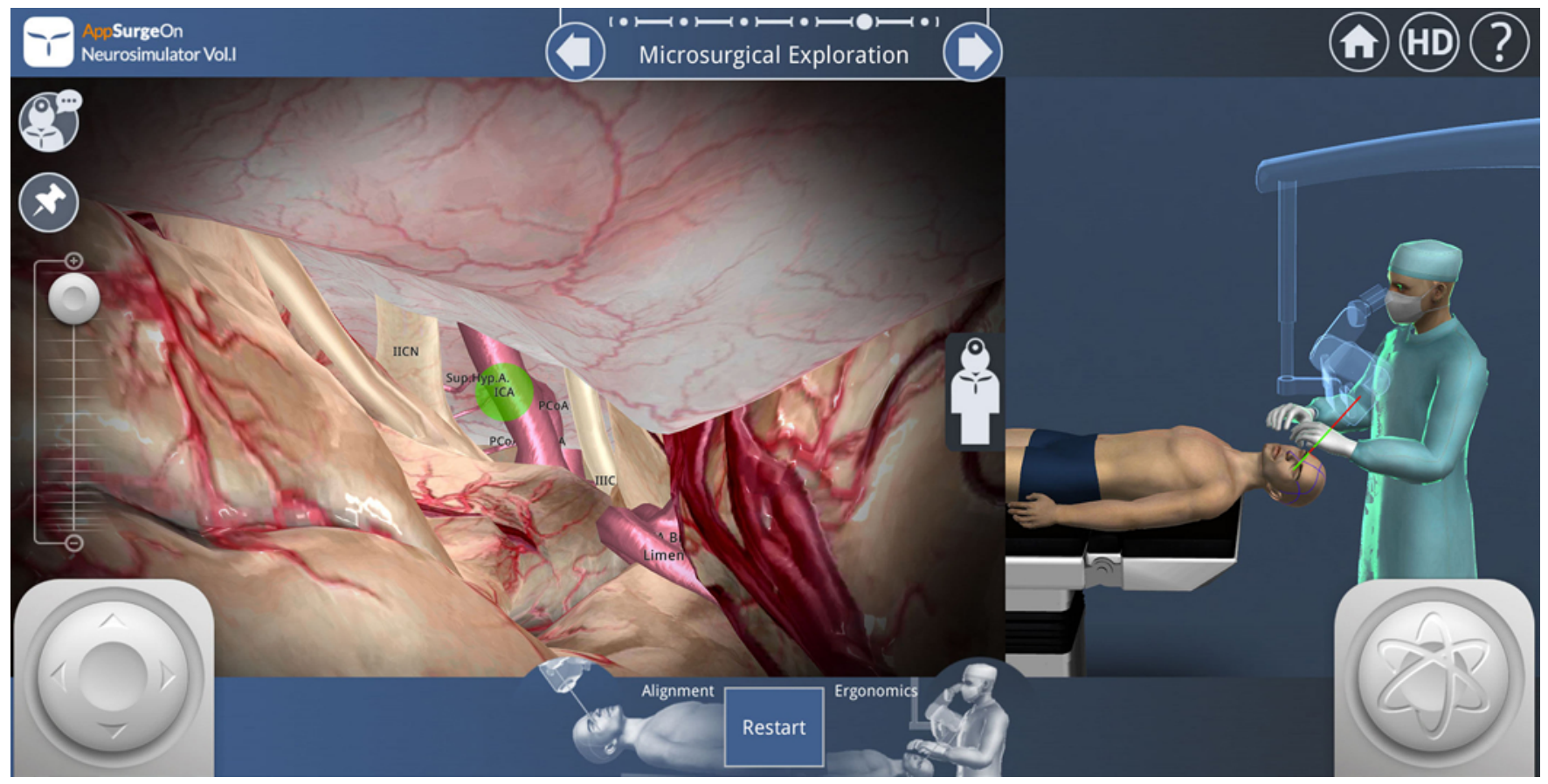

FIG. 3. Screenshot from the app UpSurgeOn. Copyright UpSurgeOn/Federico Nicolosi MD. Used with permission. 

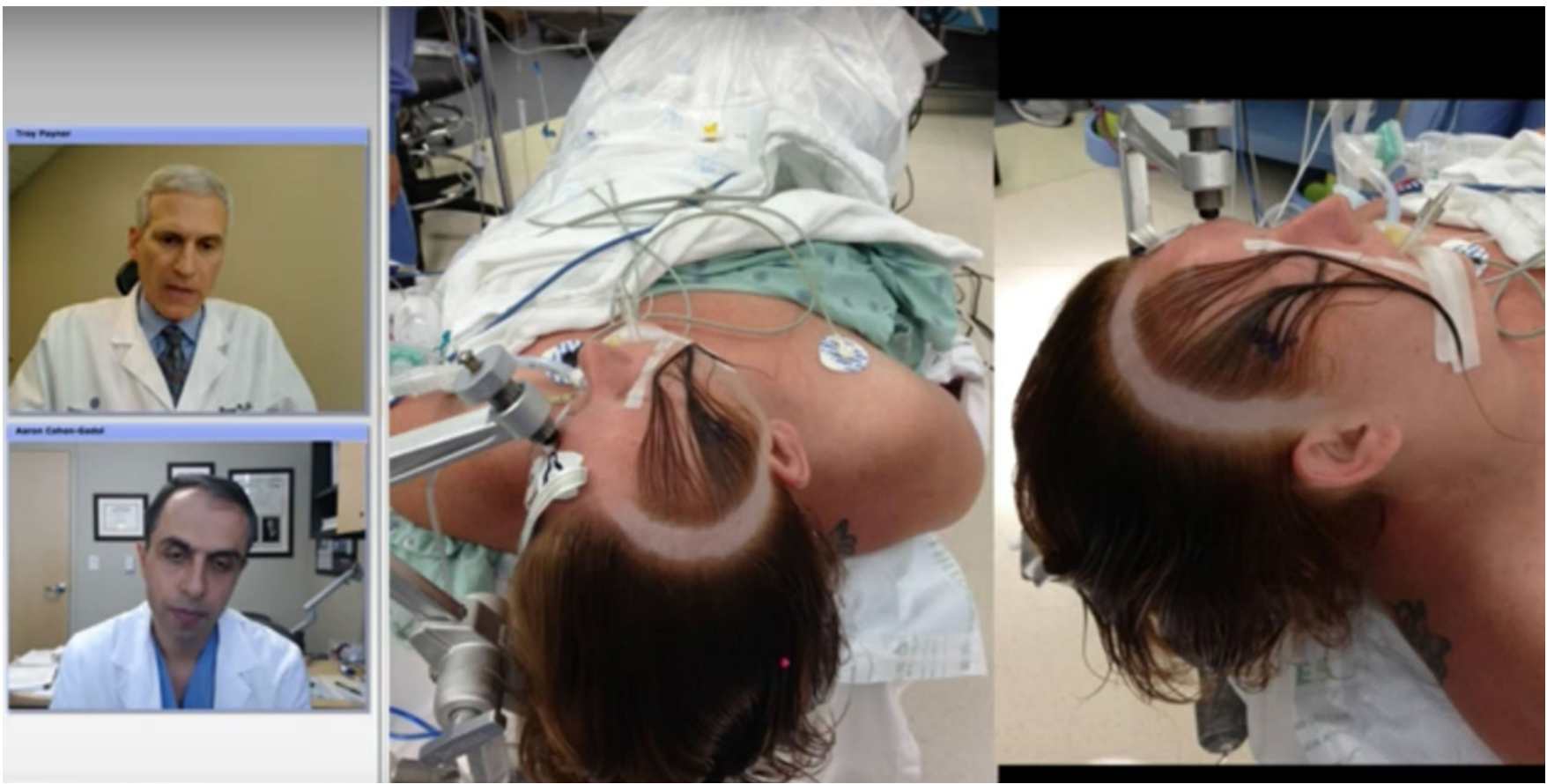

FIG. 4. Screenshot from Paynor T, Cohen-Gadol A: Pterional Craniotomy: Details from Technique. The Neurosurgical Atlas on YouTube. Retrieved from https://www.youtube.com/watch?v=vDGO4kVy0Gc. Copyright 2014 Aaron Cohen-Gadol. Used with permission.

app that provides a quick reference to assist in decisionmaking concerning and management of neurosurgical patients (www.neurosurgerysurvivalguide.com).

\section{EANS Academy}

This platform, developed by the EANS, contains several types of documents, including abstracts of congresses, webcasts of events, and interactive quizzes. Access is free for EANS members and the members of the European neurosurgical societies.

\section{Neurosurgical.TV}

Neurosurgical.TV (www.neurosurgical.tv) provides live webinars by neurosurgeons from all over the world and podcasts from neurosurgical events worldwide. It is accessible to a global community via social media and WhatsApp discussion groups (Fig. 6).

\section{D Neuroanatomy}

3D Neuroanatomy (3DN; www.3dneuroanatomy.com) is a Spain-based website providing descriptions and 3D pictures of the main neurosurgical approaches from live surgeries or cadaver lab dissections. The website also offers intraoperative videos.

\section{The Rhoton Collection}

The Rhoton Collection (www.rhoton.ineurodb.org) is an online resource for neuroanatomy and neurosurgery education. Thanks to the support of the American Association of Neurological Surgeons (AANS) and Neurosurgery Research and Education Foundation (NREF), the archive produced by Dr. Rhoton and his fellows from all over the world, as well as volunteers and artists, during about 40 years, has been made available on this website. It

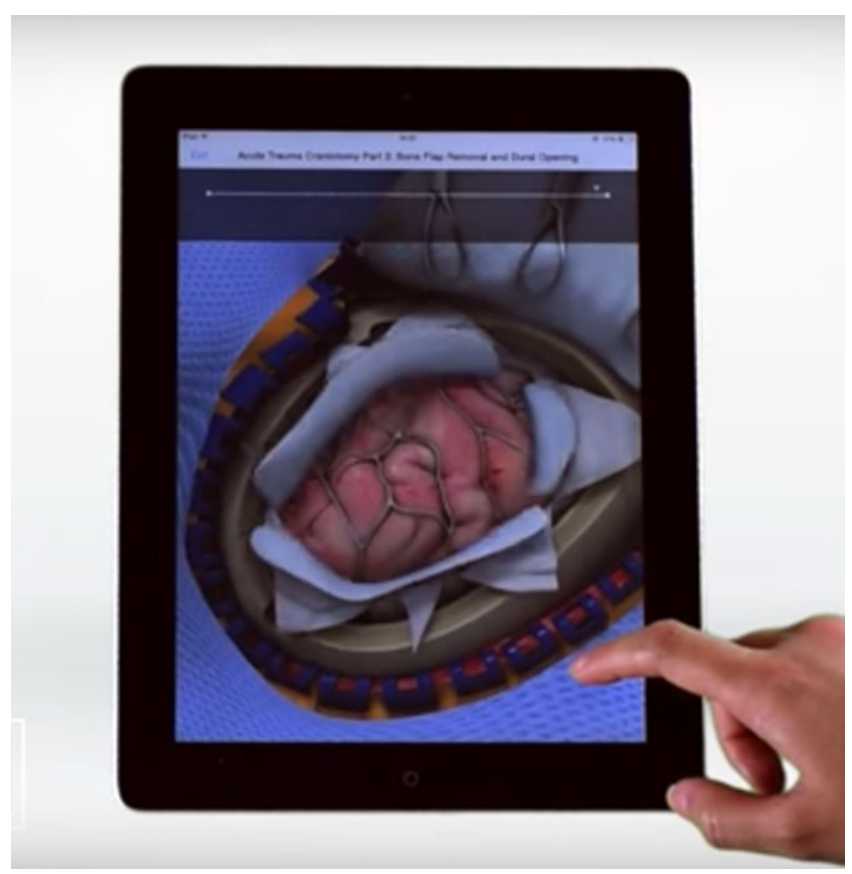

FIG. 5. Screenshot of video from Touch Surgery on YouTube. https:// www.youtube.com/channel/UCAU6QOyPVd3AIQvUinHgXhQ. Copyright Touch Surgery. Used with permission. 


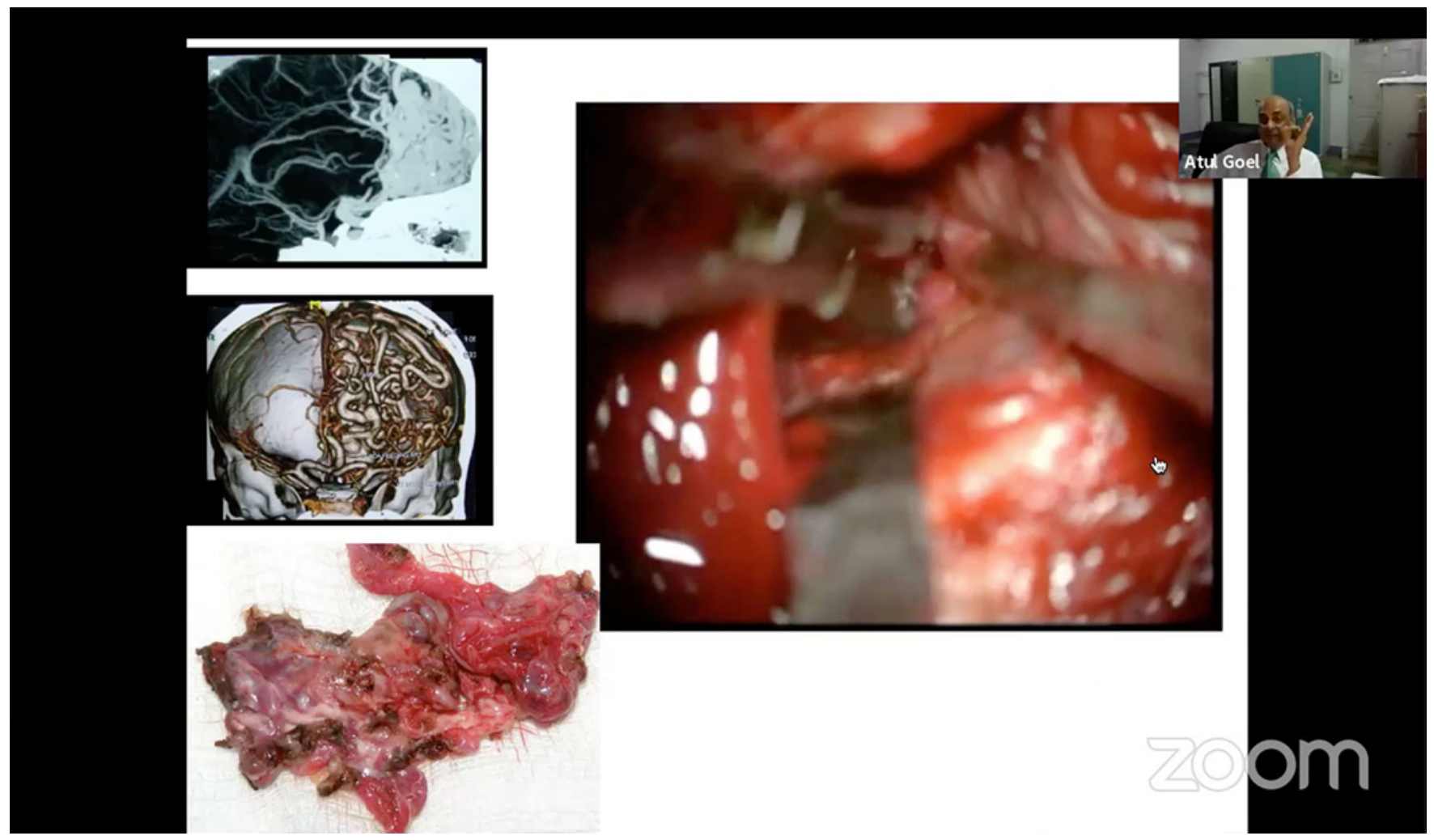

FIG. 6. Screenshot from NeuroSurgical.TV (http://www.neurosurgical.tv/). Copyright 2018 Neurosurgical.TV. OnHealthy. All Rights Reserved. Used with permission.

provides Rhoton's slides, video lectures, and an interactive atlas in 2D and 3D formats.

\section{Hinari}

The Hinari Access to Research for Health Programme (www.who.int/hinari) was created to support education and research programs in LMICs. It was set up by the World Health Organization (WHO) together with major publishers and was developed in the framework of the Health InterNetwork, introduced by United Nations (UN) Secretary General Kofi Annan at the UN Millennium Summit in the year 2000. It provides online access to one of the world's largest collections of biomedical and health literature at no or very low cost. The collection contains up to 13,500 journals (in 45 different languages), 60,000 e-books, and 110 other information resources, which are now available to health institutions in more than 115 countries, areas, and territories, benefiting many thousands of health workers and researchers, and in turn, contributing to improve world health.

\section{Discussion}

The lack of neurosurgical education has a devastating impact in terms of neurosurgical care in developing countries. This applies to any surgical field.

According to the data from the Lancet Commission on Global Surgery, $30 \%$ of diseases worldwide need surgical treatment. However, as much as $90 \%$ of the population in low-income countries in areas such as South Asia and sub-
Saharan Africa (a total of 5 billion worldwide) lacks access to basic surgical services. ${ }^{13}$ The number of neurosurgeons available to meet the major needs of the population in countries with limited facilities is still low. We find a great discrepancy between the number of neurosurgeons in developed countries ( 1 per 80,000 patients) and in large areas of the LMICs, where we found ratios of 1 to 10 million. For example, while in the United States there is 1 neurosurgeon per 62,500 people, in sub-Saharan Africa there is only 1 neurosurgeon for approximately 7 million people (https:// globalneurosurgery.org/global-neurosurgery-workforcemap). ${ }^{6}$

The implementation of a sustainable training system in these countries requires significant resources and many years. ${ }^{14,17}$

Some authors have pointed out critical issues that must be addressed in order to face the increasing demands of neurosurgical care. Such issues may be negotiated by investing in resources to improve transportation from rural areas to hospitals, minimize costs for surgical procedures, and create global networks between hospitals in LMICs and Western neurosurgical centers.

However, the majority of authors emphasize the importance of investing both funds and human resources in education. The education of people in countries with limited resources is the major contribution to the local population in creating an autonomous organization, with proper experience and research projects, gradually limiting the need for assistance from Western neurosurgeons. Exporting hu- 
man resources undoubtedly offers an efficacious service to the LMICs, but a long-term attitude can often look like colonization.

As is well known, neurosurgical education takes time and cannot be separated from training gained through reading books and journal articles, assisting in the operating theater, attending medical congresses and workshops, and practicing in cadaver labs. All these activities need a level of financial support that is seldom found by neurosurgeons in LMICs.

Education tools for widespread use in countries with limited facilities should be not only easily accessible and low cost, but also regularly updated, oriented to surgical practice, and of scientific validity (supported by international neurosurgical societies and/or journals with high impact factor).

Gonzalez et al. present their experience of providing neurosurgical residents with digital and mobile educational resources in support of the departmental academic activities at UCLA. ${ }^{8}$ They demonstrated that a structured, competency-based neurosurgical education program, supported by digital and mobile resources, improved reading habits among residents and also improved their performance on the Congress of Neurological Surgeons SelfAssessment in Neurological Surgery examination.

As widely accepted, neuroanatomy knowledge represents a cornerstone in neurosurgical education. The experience of cadaveric dissections cannot be matched by any substitute, but its cost makes it of limited accessibility in LMICs. The Rhoton collection is one of the most extensive and authoritative archives for neuroanatomy. Thanks to the support of the AANS and NREF, the results of over 40 years of dissections by Dr. Rhoton have been made available online with the goal of providing high-quality teaching materials for education.

Neurosurgical education in LMICs should be at first oriented to transferring information strictly related to surgical practice, a goal not yet pursued by common education sources. This knowledge is often transmitted through interaction and case sharing. For example, in the 100 UCLA Subjects in Neurosurgery, experts from different neurosurgical subspecialties present basic principles, current concepts, and extensive reviews as well as their own experience and developments in specific topics. Considering the importance of experience and active sharing, the EANS Academy provides abstracts of congresses, webcasts of events, and interactive quizzes, although such materials are usually only accessible to EANS members. Along a similar line, The Neurosurgical Atlas supports teaching through the participation of experts who share tips and tricks of several procedures. ${ }^{16}$ The WFNS Young Neurosurgeons Committee shares experiences through WFNS YNF webinars and other activities advertised by the AppSurgeOn Stream and through the Brainbook social media pages. Similarly, Neurosurgical.TV offers opportunities to participate in discussions with expert panelists, connecting different specialists from around in the world.

Despite the difficulty in transferring surgical skills to and from settings other than the operating theater, we believe that some digital tools that allow better understanding of neurosurgical procedures or simulate surgical sce- narios can represent an accessible way to bridge the gap in neurosurgical education between surgeons from Western countries and colleagues from LMICs.

The AppSurgeOn app series, designed by UpSurgeOn, has been designed by a team of young neurosurgeons to improve 3D imagination, visuospatial skills, and surgical anatomy knowledge through mobile platform-based interactivity. Currently the series includes only 3 apps, but an effort is underway to expand this library through research and development. With a similar vision, but more focused on procedural automatisms, the Touch Surgery platform simulates over 100 procedures from many surgical specialties. These procedures are step-by-step interactive animations that allow users to improve their understanding of a standard technique. The platform also offers tools for self-assessment, but it currently includes only 9 procedures for neurosurgery.

Another important aspect of neurosurgical digital teaching is the support of decision-making. As is well known, the gold standards of clinical management require the use of proper grading and classification systems. Supported by EANS, the NeuroMind app offers a wide library of scores, scales, and classifications, most of them designed as open-source algorithms that can calculate score values if the appropriate parameters are inserted. NeuroMind is one of most frequently downloaded neurosurgery-related apps, but it is generally useful for a more advanced audience. Along the same line, the Neurosurgery Survival Guide (NSG) may be considered a reference for many neurosurgical residents, interns, medical students, and other midlevel practitioners.

Finally, the development of neurosurgical education in these countries comes about through the effort of workers and researchers contributing directly to improve healthcare systems. In order to support research programs in more than 115 LMICs, WHO, together with major journals, promotes the Hinari project. It provides online access to journals, e-books, and other resources free of charge or at a very low price. This project was officially launched in January 2002, with some 1500 journals from 6 major publishers: Blackwell, Elsevier Science, the Harcourt Worldwide STM Group, Wolters Kluwer International Health \& Science, Springer Verlag, and John Wiley. During the subsequent years, the numbers of participating publishers has continued to increase.

Although there are numerous challenges, including lack of resources (such as computers and stable Internet access), cultural differences, and language comprehension problems, the projects dealing with neurosurgical digital content are working in the right way. The widespread lowcost dissemination of neurosurgical education is the main aim of these platforms. Limiting the differences in neurosurgical knowledge is the only way to reduce inequities in LMICs.

\section{Conclusions}

Online resources for neurosurgical education are used worldwide on a daily basis. They represent a new trend in providing education and knowledge in neurosurgery, regardless of the country's wealth. These tools can be consid- 
ered a valid educational support to bridge the gap between surgeons from high and low-middle income countries.

Further resources and digitally supported programs are desirable to promote the dissemination of neurosurgical information in these countries.

\section{Acknowledgments}

Angelos Kolias is supported by the National Institute for Health Research (NIHR) Global Health Research Group on Neurotrauma, which was commissioned by the NIHR using Official Development Assistance (ODA) funding (project 16/137/105). The views expressed in this publication are those of the author(s) and not necessarily those of the NHS, National Institute for Health Research, or the Department of Health.

\section{References}

1. Bernardo A: Virtual reality and simulation in neurosurgical training. World Neurosurg 106:1015-1029, 2017

2. Blankstein U, Dakurah T, Bagan M, Hodaie M: Structured online neurosurgical education as a novel method of education delivery in the developing world. World Neurosurg 76:224-230, 2011

3. Budohoski KP, Ngerageza JG, Austard B, Fuller A, Galler R, Haglund M, et al: Neurosurgery in East Africa: innovations. World Neurosurg 113:436-452, 2018

4. Carbonaro M, King S, Taylor E, Satzinger F, Snart F, Drummond J: Integration of e-learning technologies in an interprofessional health science course. Med Teach 30:25-33, 2008

5. Casey KM: Opening the doors to surgical education in lowand middle-income countries. JAMA Surg 149:347, 2014

6. Dewan MC, Rattani A, Fieggen G, Arraez MA, Servadei F, Boop FA, et al: Global neurosurgery: the current capacity and deficit in the provision of essential neurosurgical care. Executive Summary of the Global Neurosurgery Initiative at the Program in Global Surgery and Social Change. J Neurosurg [epub ahead of print April 27, 2018. DOI:

7. Ellegala DB, Simpson L, Mayegga E, Nuwas E, Samo H, Naman N, et al: Neurosurgical capacity building in the developing world through focused training. J Neurosurg 121:15261532,2014

8. Gonzalez NR, Dusick JR, Martin NA: Effects of mobile and digital support for a structured, competency-based curriculum in neurosurgery residency education. Neurosurgery 71:164-172, 2012

9. Jotwani P, Srivastav V, Tripathi M, Deo RC, Baby B, Damodaran N, et al: Free-access open-source e-learning in comprehensive neurosurgery skills training. Neurol India 62:352-361, 2014

10. Liang KE, Bernstein I, Kato Y, Kawase T, Hodaie M: Enhancing neurosurgical education in low- and middle-income countries: current methods and new advances. Neurol Med Chir (Tokyo) 56:709-715, 2016

11. Mashiko T, Kaneko N, Konno T, Otani K, Nagayama R, Watanabe E: Training in cerebral aneurysm clipping using self-made 3-dimensional models. J Surg Educ 74:681-689, 2017

12. Mayer RE: What neurosurgeons should discover about the science of learning. Clin Neurosurg 56:57-65, 2009
13. Meara JG, Hagander L, Leather AJM: Surgery and global health: a Lancet Commission. Lancet 383:12-13, 2014

14. Park KB, Johnson WD, Dempsey RJ: Global neurosurgery: the unmet need. World Neurosurg 88:32-35, 2016

15. Piquer J, Qureshi MM, Young PH, Dempsey RJ: Neurosurgery Education and Development program to treat hydrocephalus and to develop neurosurgery in Africa using mobile neuroendoscopic training. J Neurosurg Pediatr 15:552-559, 2015

16. Rutka JT: Editorial. Mastering the art of complex neurosurgical procedures: The Neurosurgical Atlas and the Journal of Neurosurgery. J Neurosurg 126:1029-1032, 2017

17. Santos MM, Qureshi MM, Budohoski KP, Mangat HS, Ngerageza JG, Schöller K, et al: The growth of neurosurgery in East Africa: challenges. World Neurosurg 113:425-435, 2018

18. Schirmer CM, Elder JB, Roitberg B, Lobel DA: Virtual reality-based simulation training for ventriculostomy: an evidence-based approach. Neurosurgery 73 (Suppl 1):66-73, 2013

19. Servadei F, Rossini Z, Nicolosi F, Morselli C, Park KB: The role of neurosurgery in countries with limited facilities: facts and challenges. World Neurosurg 112:315-321, 2018

20. Spetzler RF, Kick SA: The status of neurosurgery in the United States: 2010 and beyond. World Neurosurg 74:3240, 2010

21. Stienen MN, Netuka D, Demetriades AK, Ringel F, Gautschi OP, Gempt J, et al: Neurosurgical resident education in Europe-results of a multinational survey. Acta Neurochir (Wien) 158:3-15, 2016

22. Stienen MN, Schaller K, Cock H, Lisnic V, Regli L, Thomson $\mathrm{S}$ : eLearning resources to supplement postgraduate neurosurgery training. Acta Neurochir (Wien) 159:325-337, 2017

\section{Disclosures}

Dr. Nicolosi reports an ownership relationship with UpSurgeOn and a nonfinancial (personal) relationship with UpSurgeOn. Dr. Servadei reports that he is the president of the WFNS.

\section{Author Contributions}

Conception and design: Rossini, Nicolosi, Servadei. Acquisition of data: Rossini, Nicolosi, Zaed. Analysis and interpretation of data: Rossini, Nicolosi, Kolias. Drafting the article: Rossini, Nicolosi, Servadei. Critically revising the article: Rossini, Nicolosi, Kolias, Fornari, Servadei. Reviewed submitted version of manuscript: Rossini, Nicolosi, Kolias, Fornari, Servadei. Approved the final version of the manuscript on behalf of all authors: Rossini. Administrative/technical/material support: Nicolosi, Servadei. Study supervision: Rossini, Nicolosi, Servadei.

\section{Correspondence}

Zefferino Rossini: Humanitas Research Hospital, Rozzano, Italy. zefferino.rossini@unimi.it. 